\title{
A Reserve Planning Method of Transformer Group based on Markov Reliability Model
}

\author{
Yuan Tian ${ }^{1}$, Hongliang Liu ${ }^{1}$, Zhiyong $\mathrm{Chen}^{2}$, Huan $\mathrm{Li}^{2}$ \\ ${ }^{1}$ State Grid Hebei Electric Power Co., Ltd. Electric Power Research Institute, Shijiazhuang, 050021, China \\ ${ }^{2}$ PINGGAO GROUP CO.,LTD, Pingdingshan, Henan 467001, China
}

Received: June 15, 2021. Revised: December 4, 2021. Accepted: January 10, 2022. Published: January 12, 2022.

\begin{abstract}
In view of the current situation that the load difference is not considered in the existing research on public standby transformer, the reliability Markov model and public standby planning model of distribution transformer unit based on public standby mode are proposed. When the transformer fails, the standby transformer is preferred to replace the faulty transformer. The power failure time of the user is shortened from the transformer maintenance time to the standby installation and operation time. The state transition rate is the installation and operation rate of the standby transformer, and the replaced faulty transformer is converted to the standby transformer in the unavailable state. This paper applies the scheme decision analysis method to the field of engineering control, and applies the combination of the compound matter element analysis and the subjective and objective weight to the quantitative calculation of correlation entropy. This method solves the current situation that transformer faults are difficult to measure and calculate quantitatively, and it can accurately predict the healthy development trend of transformers, which has a good guiding value for the use and maintenance of transformers. At the same time, the public standby measures of distribution transformer can better solve the problem of long transformer fault repair time with a small amount of capital investment, effectively shorten the recovery time of fault outage and reduce the impact of transformer fault. The standby measures of distribution transformer have an obvious impact on the reliability of distribution system.
\end{abstract}

Keywords:- Reserve Planning Method, Transformer Group, Markov Reliability Model, Health Degree

\section{INTRODUCTION}

A $\mathrm{s}$ the key equipment of distribution system, distribution transformer takes a long time to repair its fault and has a great impact of power failure[1]. The public standby measures of distribution transformer can better solve the problem of long transformer fault repair time with a small amount of capital investment, effectively shorten the recovery time of fault outage and reduce the impact of transformer fault. The standby measures of distribution transformer have an obvious impact on the reliability of distribution system [2-3]. Reference [4-6] analyzes the common standby measures of distribution transformers from the perspective of reliability. Among them, Reference [4] uses Markov method to model the reliability of distribution transformer groups with common standby, but this study does not take into account the differences between transformers. In reference [5], the state enumeration method is used to calculate the failure probability of transformer unit based on equipment aging failure, and the input strategy of standby equipment is determined based on this criterion. Reference [6] considers the standby settings under two fault modes of transformer repair failure and irreparable failure, and takes the system average outage duration index as the criterion to formulate the standby equipment investment plan in the planning cycle.

Reference [7] analyzes the influence of equipment aging on fault rate in combination with statistical data, and establishes a standby optimization model aiming at the sum of investment cost and operation cost of standby transformer. Based on the probability distribution model of distribution transformer group based on Poisson distribution, Reference [8] proposed the minimum cost criterion of optimal reserve, and determined the optimal reserve quantity under each criterion. Reference [9] uses sequential Monte Carlo simulation method to calculate the probability and frequency index of distribution transformer group with standby equipment, and establishes the standby optimization model, but the model does not consider the influence of the installation and operation process of standby transformer. Reference [10] proposed a calculation method of common reserve quantity of distribution transformer unit based on probability criterion, and analyzed the changes of investment cost and outage loss cost under different reserve quantities.

The above literature studies the optimal standby mode and quantity of distribution transformer units from the perspective of economy and reliability, but there are few studies on the public standby of distribution transformer units considering both reliability and load difference, and few studies consider the priority of installation and operation of standby transformers. Taking the equipment group composed of distribution transformers with one or several outgoing lines in a region as the research object, considering the differences in the importance and size of distribution transformer load, this paper analyzes the state transfer process and transfer rate of distribution transformer group, establishes the reliability 
Markov model of distribution transformer group, and then establishes the public standby optimization planning model based on economy.

\section{RELIABILITY MARKOV MODEL BASED ON STANDBY TRANSFORMER GROUP}

\section{A. Markov Model}

There is a phenomenon in real life, that is, in the case of the known current conditions, situations occurring in the future are only related to the present instead of the past events, and the model describing the phenomenon is Markov chain model. Assume $\left\{\xi_{n}, n=1,2, \cdots\right\}$ is random sequence, state space $E$ is finite or countable set, corresponding to arbitrary positive integers $m, n$, and if $i, j, i_{k} \in E(k=1, \cdots, n-1)$, $P\left\{\xi_{n+m}=j \mid \xi_{n}=i, \xi_{n-1}=i_{n-1}, \cdots, \xi_{1}=i_{1}\right\}=P\left\{\xi_{n+m}=j \mid \xi_{n}=i\right\}$

Thus, $\left\{\xi_{n}, n=1,2, \cdots\right\}$ is called as a Markov chain, Equation (1) is called as Markov property. Assume $\left\{\xi_{n}, n=1,2, \cdots\right\}$ is a Markov chain. If the right conditional probability of Equation (1) is unrelated to $n$,

$$
P\left\{\xi_{n+m}=j \mid \xi_{n}=i\right\}=P_{i j}(m)
$$

Thus, $\left\{\xi_{n}, n=1,2, \cdots\right\}$ is called as Markov chain of time-homogeneity. $P_{i j}(m)$ is the transition probability of system transferring from state $i$ to state $j$ through $m$ time intervals (or $m$ steps). Equation (2) is called as time-homogeneity.

For a Markov chain $\left\{\xi_{n}, n=1,2, \cdots\right\}$, matrix $P(m)=\left(P_{i j}(m)\right)$ whose element is $m$ step transition probability. When $m=1, P(1)$ is simply transfer matrix. They have three basic properties

(1) For any $i, j \in E, \quad 0 \leq p_{i j}(m) \leq 1$;

(2) For any $i \in E, \sum_{j=E} p_{i j}(m)=1$

(3) For any $i, j \in E, \quad p_{i j}(0)=\delta_{i j}=\left\{\begin{array}{l}1, i=j \\ 0, i \neq j\end{array}\right.$

When the actual problem can be described with Markov chain, we first need to determine its state space and parameter set, and then determine its one-step transition probability.

\section{B. Reliability Markov Model of Transformer Group}

The state of distribution transformer group is discrete in space and continuous in time. It is in a discrete state at a certain time until it is transferred to another discrete state. Markov model can clearly show the state and mutual transfer relationship of transformer group, so as to calculate the probability and frequency index of transformer group in each state.

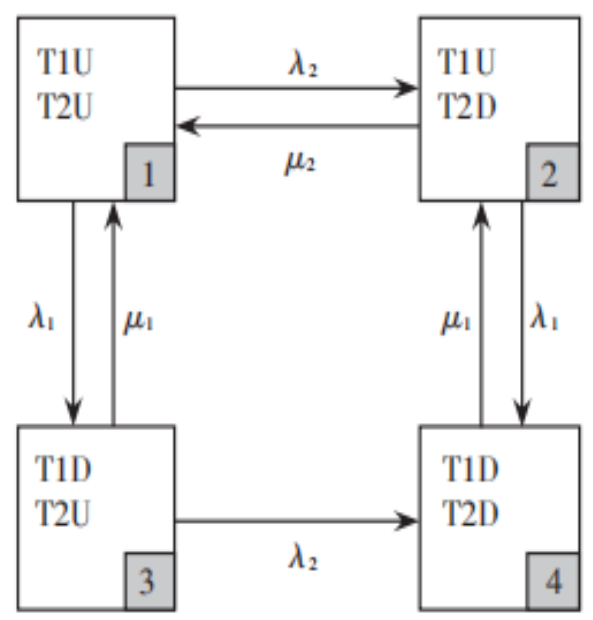

Fig. 1 Markov state space of Transformer Group

In the case of excluding public standby, the state of transformer is operation and fault, and the transfer between States is composed of fault transfer and repair transfer. When multiple transformers fail at the same time, priority shall be given to the maintenance of transformers with greater power failure loss. In order to illustrate the modeling process of reliability Markov model, a transformer group composed of two transformers is taken as an example. The state space of the system is shown in Figure 1.

In Figure1, $U$ and $D$ respectively indicate that the distribution transformer is in operation and fault state. $\lambda_{I}$ and $\mu_{I}$ is the failure rate and repair rate of distribution transformer respectively. T1 and T2 are transformer numbers and the number in the lower right corner of the box is the status number.

It can be seen from Figure 1 that when there is no transformer fault, it will be transferred through fault transfer. When only one transformer fails, repair the faulty transformer from state 2 and state 3 to state 1 or from fault transfer to state 4 . When two transformers fail, priority will be given to repair transformer $\mathrm{T} 1$ and transfer to state 2 .

The total number of states in the state space diagram is related to the number of transformers and the number of transformer states. The total number of states of the equipment group composed of $\mathrm{N} 2$-state transformers is $2^{n}$. In order to reduce the computational complexity, high-order fault events are omitted to reduce the total number of system states. The calculation formula of the total number of states is

$$
S_{T}=C_{N}^{0}+C_{N}^{1}+\cdots+C_{N}^{D}=\sum_{d=0}^{D} C_{N}^{d}
$$

Where, $S_{T}$ is the total number of states, $d$ is the fault order, and $D$ is the highest fault order (2 or 3 ).

\section{Reliability Markov Model of Common Standby} Transformer Group

For the transformer bank with public standby, the state of the public standby transformer and its installation, operation and transfer process shall be considered in the state space diagram. The status of standby transformer is divided into 
available status and unavailable status, in which: available status refers to the status that can be installed and put into operation; Unavailable status refers to the status that can be installed and put into operation only after repair.

When the transformer fails, the standby transformer is preferred to replace the faulty transformer. The power failure time of the user is shortened from the transformer maintenance time to the standby installation and operation time. The state transition rate is the installation and operation rate of the standby transformer, and the replaced faulty transformer is converted to the standby transformer in the unavailable state. If two or more transformers fail at the same time, priority shall be given to replace or repair the transformer with greater power failure loss.

During the analysis, the following assumptions are made. (1) When the standby transformer is idle and available, it is $100 \%$ reliable. (2) In case of transformer failure, spare replacement measures shall be taken. (3) When multiple transformers fail at the same time, standby replacement or maintenance shall be carried out according to the outage loss. (4) After the standby replacement operation is completed, start to repair the faulty transformer. (5) Only one faulty transformer can be repaired or installed and put into operation at a time, and the fault rate is the same before and after updating. (6)The limitations of maintenance resources and maintenance personnel are not considered in the model. A Markov state space of a distribution transformer bank with one standby transformer is shown in Figure 2.

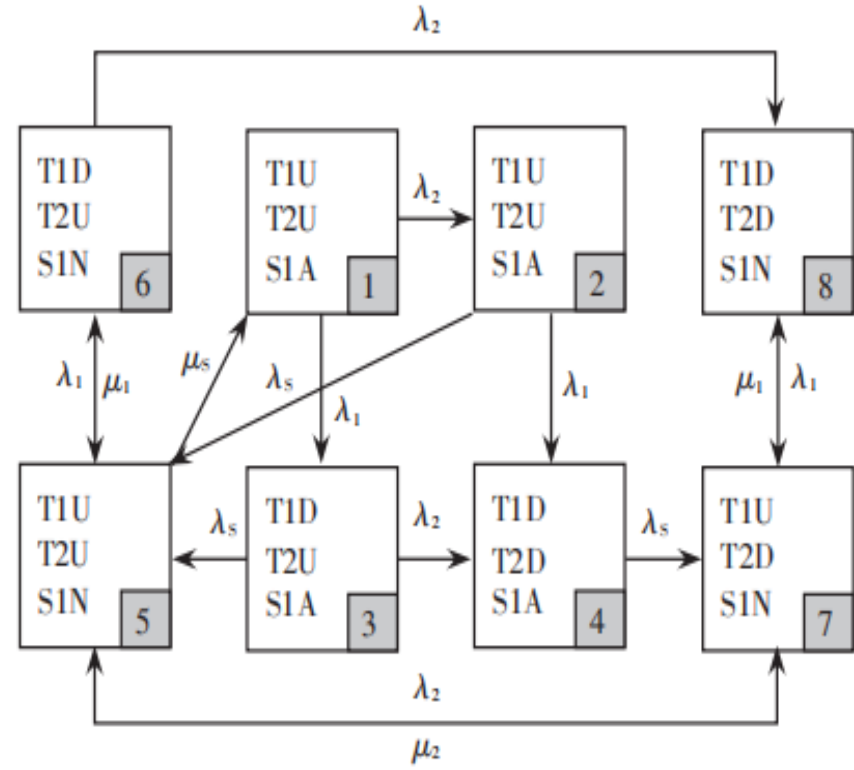

Fig. 2 Markov state space of common standby transformer group

In Figure 2, $S_{1}$ is the number of standby transformer, $A$ indicates that the standby transformer is available, which can be directly used to replace the fault transformer, $N$ indicates that the standby transformer is unavailable, which can be used to replace the fault transformer after repair, $\lambda_{s}$ is the installation and operation rate and $\mu_{s}$ is the repair rate of public standby transformer.

It can be seen from Figure 2 that when there is a transformer fault, the standby transformer is preferred to replace the fault transformer to shorten the power outage time. For example, in state 3, transformer T1 fails and standby transformer S1 is available, so standby transformer is used to replace $\mathrm{T} 1$ and transfer to state 5 , and the transfer rate is the installation and operation rate of standby transformer; In state 5 , the standby transformer is in the unavailable state. After repair, it will be converted to the standby transformer in the available state, that is, it will be transferred to state 1 , and the transfer rate is the repair rate of the standby transformer. After considering standby, the number of states of the system is much more than that without standby, and the number of States will rise rapidly with the increase of the number of transformers and standby. Compared with the conventional transformer, the standby transformer is not connected to the load and there is no load difference, so the standby transformer is not distinguished.

Only the state where the number of faulty or unavailable equipment is less than or equal to $D$ is considered, that is, the number of transformers in fault state and the number of standby transformers in unavailable state are $d$ at most. The maximum fault order $D$ is required to be greater than or equal to the number of standby transformers $S$. Then, when the system contains $\mathrm{s}$ standby transformers, the calculation formula of the total number of states is

$$
\begin{aligned}
& S_{T}=\left(C_{N}^{0}+C_{N}^{1}+\cdots+C_{N}^{D}\right)+\left(C_{N}^{0}+C_{N}^{1}+\cdots+C_{N}^{D-1}\right) \\
& +\cdots+\left(C_{N}^{0}+C_{N}^{1}+\cdots+C_{N}^{D-S}\right)=\sum_{s=0}^{S} \sum_{d=0}^{D-s} C_{N}^{d}
\end{aligned}
$$

\section{CALCULATION OF PROBABILITY AND FREQUENCY INDEX OF DISTRIBUTION TRANSFORMER GROUP BASED ON RELIABILITY MARKOV MODEL}

According to the Markov state space diagram of transformer group, the limit probability of transformer group in each state can be obtained. The state random transition rate matrix is the key to calculate the limit state probability. For Markov processes with state number st, the state random transition rate matrix $T$ is a square matrix of $S_{T}$ order. According to the definition of limit state of Markov process, the probability of limit state remains unchanged in the further transfer process, and the following formula can be obtained

$$
P T=P
$$

Where $P=\left[P_{1}, P_{2}, \ldots, P_{S T}\right]$ is the row vector of limit state probability.

Because the sum of the probabilities of all system states is 1, the formula (6) can be obtained

$$
\sum_{i=1}^{S_{T}} P_{i}=[1,1, \cdots, 1] P^{T}=1
$$

Combining equation (5) and equation (6), the limit state probability of transformer group can be obtained. Because the transformer fault will lead to load loss of power, the load loss probability of transformer $n$ is calculated by the limit state probability of transformer group. 


$$
P_{n}=\sum_{i \in S_{T n}} P_{i}
$$

Where $S_{T n}$ is the set of load loss states of transformer $n$ in the state space diagram, and $P_{n}$ is the load loss probability of transformer $n$.

The frequency of each state and the frequency of transition between states are calculated by combining Markov state space diagram and random transition rate matrix.

$$
\left\{\begin{array}{l}
f_{i}=P_{i}\left(1-T_{i i}\right) \\
f_{i j}=P_{i} T_{i j}
\end{array}\right.
$$

Where $f_{i}$ and $f_{i j}$ are the occurrence frequency of state $i$ and the frequency from state $i$ to state $j$ respectively. $T_{i i}$ and $T_{i j}$ are the transfer rates of state $i$ to state $i$ and state $j$, respectively. The loss of load frequency of transformer $n$ is

$$
f_{n}=\sum_{i \in S T_{n}} f_{i}-\sum_{i, j \in S T_{n}} f_{i j}
$$

Similar to the calculation of load loss frequency, the calculation formula of installation and operation frequency of standby transformer is

$$
f_{s}=\sum_{y_{i}>y_{j}} P_{i} f_{i j}
$$

Where, $y_{i}$ is the number of available standby transformers in state $i, y_{i}>y_{j}$ indicates that the number of available standby transformers of the system is reduced during the transition from state $i$ to state $j$, that is, the process is the process of standby installation and operation.

\section{PREDICTION METHOD OF RUNNING STATUS OF TRANSFORMER GROUP}

How to predict the running status of the transformer by using the calculation results of the health degree of the historical transformer, this paper uses parameter optimization to carry out the Markov modeling prediction.

The data of 48 groups is measured and the data tested in the first three weeks of each month of the year consists of three groups. Each group has a training set with 12 samples. However, 12 samples tested in the last week of each month are taken as a test set, as shown in Fig.3.

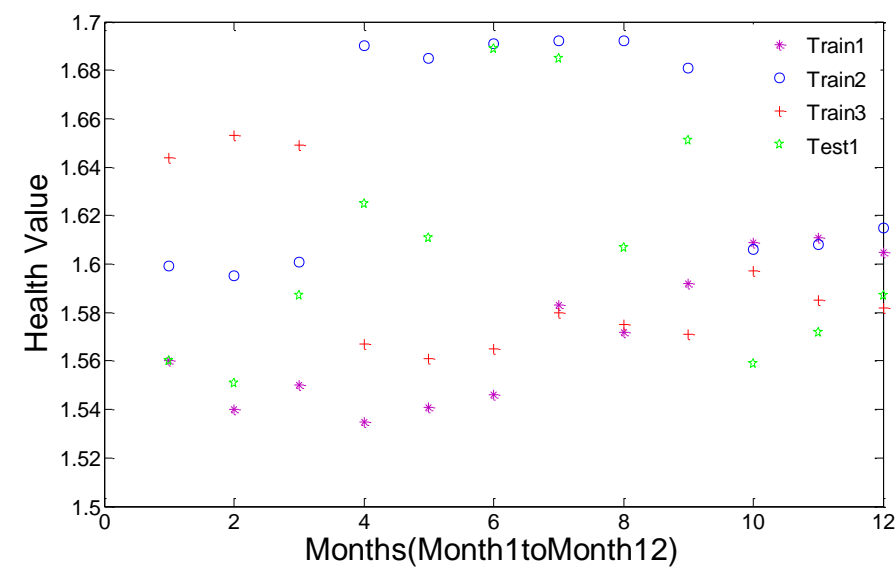

Fig. 3 Samples tested in the last week of each month are taken as a test set

The calculation and analysis of transformer's health degree are helpful for judging the current working condition of transformer. In order to use transformer more scientifically, we need to accurately predict the future operating conditions of the transformer. This paper uses parameter optimization to carry out the Markov modeling prediction, uses photoacoustic spectroscopy to test the transformer analyzed in the upper section, and regularly tests the gas produced by the transformer every week. The data tested in the first, second and third weeks of each month of the year consists of three groups. Each group has a training set with 12 samples.

This paper selects two functions: epsilon-SVR and Markov, adopts RBF kernel function, uses grid-search algorithm, GA and PSO algorithm to train the test set to carry out the parameter optimization, and also constructs the prediction model of transformer health degree. The best model is the model with the highest prediction fitting degree, and the best penalty parameter $C$ and $g$ value are determined. The specific comparison data is shown in Table 1.

Table 1. The specific comparison data

\begin{tabular}{llll}
\hline \multirow{2}{*}{ Performance } & \multicolumn{4}{l}{ Epsilon-SVM/ RBF (-s 3 -p 0.01) } \\
\cline { 2 - 4 } & Grid-search & $\mathrm{GA}$ & $\mathrm{PSO}$ \\
\hline Optimal & $\mathrm{c}=30.213$ & $\mathrm{c}=1.21$ & $\mathrm{c}=1.56$ \\
parameters & $\mathrm{g}=1.9159$ & $\mathrm{~g}=138.26$ & $\mathrm{~g}=143.24$ \\
Train-MSE & 0.0534 & 0.0044 & 0.0043 \\
Train-R & $92.54 \%$ & $95.20 \%$ & $95.23 \%$ \\
Test-MSE & 0.0534 & 0.00403 & 0.00391 \\
Test-R & $92.54 \%$ & $96.99 \%$ & $97.05 \%$ \\
\hline
\end{tabular}

The specific comparison data for Markov is shown in Table 2 . 
Table 2. The specific comparison data for Markov

\begin{tabular}{llll}
\hline \multirow{2}{*}{ Performance } & \multicolumn{2}{l}{ Markov $(-\mathrm{s} 4-\mathrm{n} 0.5)$} \\
\cline { 2 - 4 } & Grid-search & $\mathrm{GA}$ & $\mathrm{PSO}$ \\
\hline Optimal & $\mathrm{c}=30.863$ & $\mathrm{c}=2.793$ & $\mathrm{c}=1.493$ \\
parameters & $\mathrm{g}=1.9159$ & $\mathrm{~g}=145.08$ & $\mathrm{~g}=176.32$ \\
Train-MSE & 0.0534 & 0.00430 & 0.00432 \\
Train-R & $92.54 \%$ & $95.28 \%$ & $95.4 \%$ \\
Test-MSE & 0.0534 & 0.00356 & 0.00408 \\
Test-R & $92.54 \%$ & $97.17 \%$ & $96.91 \%$ \\
\hline
\end{tabular}

Table1 and Table 2 show that the prediction effect of two models. One is the model of epsilon-SVM-RBF- PSO parameters optimized by particle swarm optimization, which is called ERPSO model for short. The fitting error and the correlation coefficient are $0.0043 / 95.23 \%$, and the prediction error and the correlation coefficient are $0.00430 / 95.28 \%$. The other is the model of nu-SVM- RBF-GA parameters optimized by GA, which is called NRGA model for short. The fitting error and the correlation coefficient are $0.0043 / 95.23 \%$, and the prediction error and the correlation coefficient are $0.00356 / 97.17 \%$. The simulation curves of the two algorithms are shown in Fig.4-7.

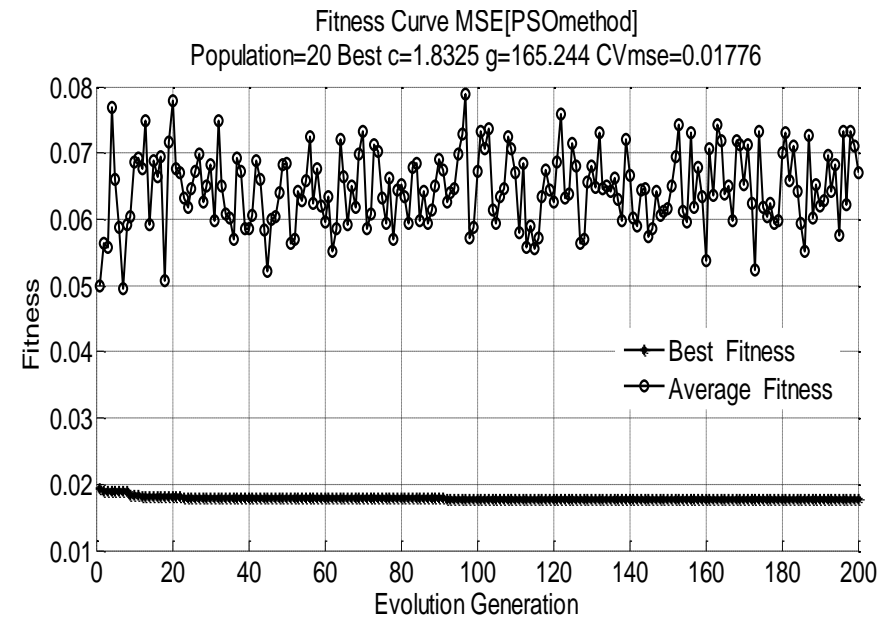

Fig. 4 Fitness curve based on ERPSO model

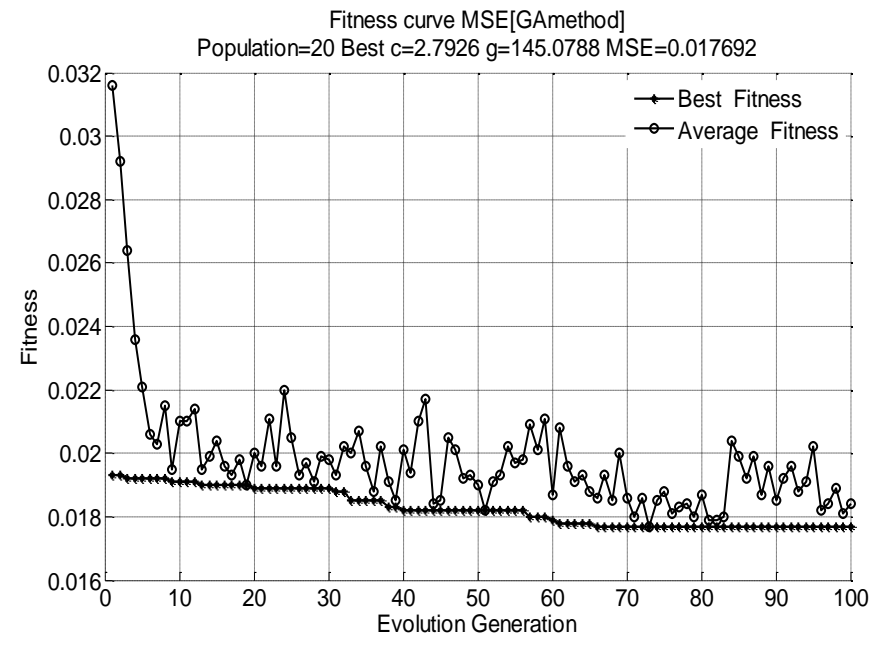

Fig. 5 Fitness curve based on Markov model

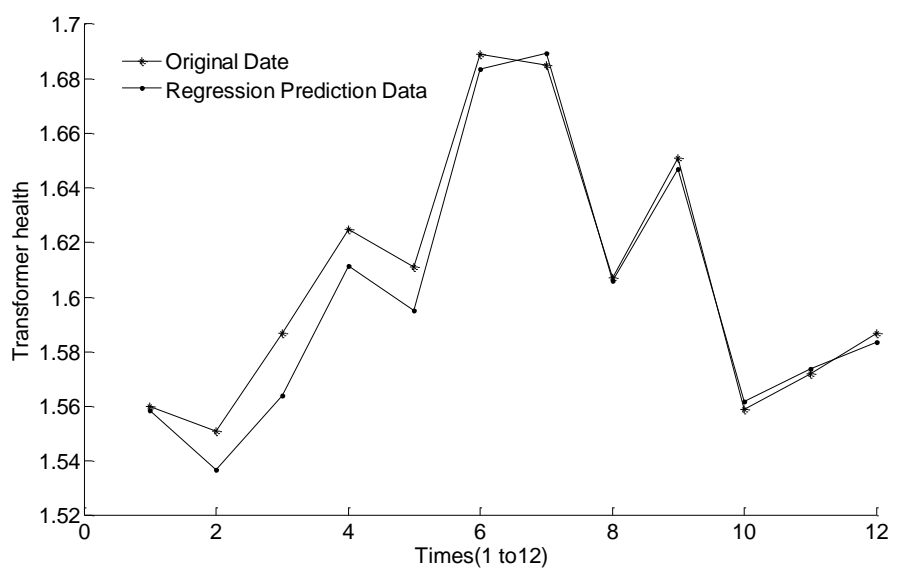

Fig. 6 Forecasting set regression forecasting curve of Transformer health based on ERPSO model

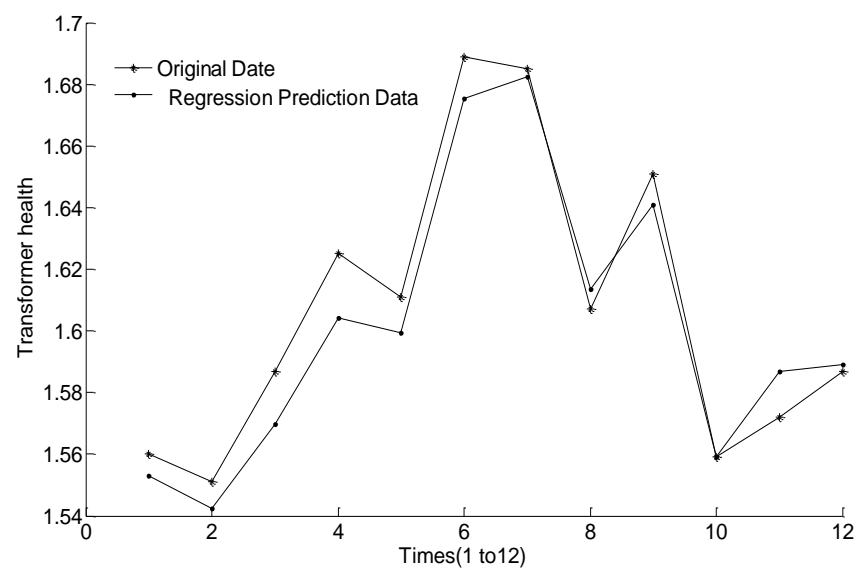

Fig. 7 Forecasting set regression forecasting curve of Transformer health based on Markov model

\section{CONCLUSION}

In this paper, the reliability Markov model of distribution 
transformer group is established, which can take into account the load difference of distribution transformer. On this basis, the public standby optimization model is established.

This paper applies the scheme decision analysis method to the field of engineering control, and applies the combination of the compound matter element analysis and the subjective and objective weight to the quantitative calculation of correlation entropy.

In future, BP algorithm can be used to optimize the fuzzy clustering analysis and prediction of transformer group, and good results are achieved. However, from the in-depth study and practical application, there are still many aspects to be further studied in the future. In the follow-up study, the clustering method will be further improved, more effective data features will be extracted, and the data mining technology will be embedded into transformer group, so as to improve the work efficiency of relevant managers and make up for the limitations of traditional analysis methods.

\section{ACKNOWLEDGEMENTS}

This work is supported by the Science and Technology Project of State Grid Hebei Electric Power Co., Ltd. (kjcb2020-012) "Structural optimization and Application Research of vehicle mounted compact mobile substation"

\section{REFERENCES}

[1] Wang T , Cai J , Meng Y, et al. A reliability evaluation method for embryonic cellular array based on Markov status graph model. Journal of Systems Engineering and Electronics, 2020, 31(2):432-446.

[2] Ma Y, Jia X, Hu Q, et al. A new state recognition and prognosis method based on a sparse representation feature and the hidden semi-Markov model. IEEE Access, 2020, PP(99):1-1.

[3] Yy A, Ieg A, Jmp B, et al. MINLP Model for Reliability Optimization of System Design and Maintenance Based on Markov Chain Representation. Computer Aided Chemical Engineering, 2020, 48:1057-1062.

[4] Zhao Y, Adjallah K H, Sava A, et al. MaxEnt feature-based reliability model method for real-time detection of early chatter in high-speed milling. ISA Transactions, 2020.

[5] Liu X , Bu T . Reliability of variable slope system and human motion image detection based on Markov chain. Arabian Journal of Geosciences, 2021, 14(11).

[6] Fma B , Wka C . Proposed Model to Intelligent Recommendation System based on Markov Chains and Grouping of Genres - ScienceDirect. Procedia Computer Science, 2020, 176:868-877.

[7] Hao Z, Maio F D, Zio E, et al. Multi-State Reliability Assessment Model of Base-Load Cyber-Physical Energy Systems (CPES) during Flexible Operation Considering the Aging of Cyber Components. Energies, 2021, 14.

[8] Zeng J J , Duan Y , Wang D, et al. Generalization performance of Lagrangian support vector machine based on Markov sampling. Journal of Statistical Planning and Inference, 2020, 214.

[9] V. Lyandres, On the Correlation Function of an Arbitrary Distributed Continuous Markov Process,
WSEAS Transactions on Circuits and Systems, Volume 19, 2020, Art. \#12, pp. 105-110.

[10] Yan L, Zhang T, Gao Y, et al. Reliability analysis of station autonomous computer system based on fuzzy dynamic fault tree and Markov model. Engineering Reports, 2021.

\section{Creative Commons Attribution License $\mathbf{4 . 0}$} (Attribution 4.0 International, CC BY 4.0)

This article is published under the terms of the Creative Commons Attribution License 4.0

https://creativecommons.org/licenses/by/4.0/deed.en_US 